\title{
Osteosynthese des Ober- und Unterschenkels mittels eingeschobener Platte
}

\author{
E. Gautier, C. B. Marti, A. J. Schuster, S. W. Wachtl, R. P. Jakob
}

\section{Zusammenfassung}

Bei der Versorgung dislozierter Unteroder Oberschenkelfrakturen stellt die minimal invasive Plattenosteosynthese eine technisch machbare Alternative zur Marknagelung dar. Die Analyse der Ergebnisse und Komplikationen nach konventioneller Plattenosteosynthese haben diesen Wandel in Bezug auf die Operationstechnik und die Art der Reposition sowie Veränderungen des Plattendesigns bewirkt. Bei der minimal invasiven subkutanen oder submuskulären Plattentechnik erfolgt die Reposition geschlossen durch manuellen oder instrumentellen Zug und die Stabilisa- tion durch eine eingeschobene LCDCP oder Kondylenplatte. Der Vorteil einer derartigen Methode liegt in der minimalen Exposition der Frakturzone, so dass die Fragmente in ihrem Weichteilverbund integriert bleiben und der natürliche Knochenheilungsprozess so wenig wie möglich gestört wird. Seit 1997 haben wir am Kantonsspital in Fribourg (Schweiz) 20 Oberschenkelfrakturen und 25 Unterschenkelfrakturen mit eingeschobener Osteosyntheseplatte stabilisiert. Trotz der neuen und damit schwierigeren Osteosynthesetechnik lag die Erfolgsrate (d.h. Knochenheilung in achsengerechter Stellung nach nur einer Operation) bei über $90 \%$.

\section{Einleitung}

Die initial erarbeiteten Prinzipien und Osteosyntheseverfahren der Arbeitsgemeinschaft für Osteosynthesefragen (AO) betonten in erster Linie mechanische Aspekte der operativen Frakturbehandlung. Eine hohe Osteosynthesestabilität wurde als notwendig erachtet, um während der Nachbehandlungsphase eine uneingeschränkte Mobilisation der Gelenke und eine Teilbelastung des verletzten Skelettabschnittes zu ermöglichen [13].

Die Grundlagenforschung der frühen 1980er Jahre aus dem Labor für experimentelle Chirurgie in Davos unter der Leitung von Prof. Perren hat zeigen können, dass jedes Implantat (sei es intramedullär oder extramedullär) zu einer teils ausgedehnten Störung der Blutversorgung des Knochens führt. Als Folge der Durchblutungsstörung entsteht eine par-

OP-JOURNAL 2000; 16: 260-267

(c) Georg Thieme Verlag Stuttgart · New York tielle Knochennekrose, welche den natürlichen Heilverlauf behindert und verzögert $[3,5,8,10,15]$.

Bei der offen durchgeführten herkömmlichen Plattenosteosynthese bewirkt die chirurgische Exposition der Fraktur, die Darstellung und Reposition der Fragmente einen nicht unerheblichen weiteren vaskulären Schaden. Chirurgische und implantatinduzierte Devaskularisation summieren sich mit dem traumatisch bedingten vaskulären Schaden des Knochens, welcher während des Unfallereignisses durch die Fragmentation des Knochens entsteht, sehr oft in unheilvoller Weise. Bekannte Komplikationen der operativen Frakturversorgung wie tiefe Infektion, verzögerte Heilung, Pseudarthrose oder Refraktur nach Entfernung des Osteosynthesematerials gehen zumindest teilweise auf das Konto des traumatisch und chirurgisch herbeigeführten Schadens der Knochen- und Weichteildurchblutung $[1,4]$.

Da die traumatisch bedingte Devaskularisation des Knochens nicht beeinflusst werden kann, gilt es, den vaskulären Schaden durch die Operation und das Implantat möglichst klein zu halten. Die Erkenntnis, dass Implantate und Fragmentmanipulation zu einer oft entscheidenden Devitalisierung des Knochens führen, hat zu einer Neuordnung der Prioritäten bei der operativen Frakturversorgung geführt - von der fast ausschließlich mechanischen Betrachtungsweise mit der Osteosynthesestabilität als wichtigstem Element hin $\mathrm{zu}$ einer biologischen Betrachtungsweise mit dem Erhalten des natürlichen Reaktionsmusters des Knochens als wichtigstem Element $[2,4,6,17,18]$. Dabei gilt es festzuhalten, dass kortikaler Knochen wesentlich empfindlicher auf einen vaskulären Schaden reagiert als der spongiöse Knochen.

Damit ist bei dislozierten Gelenkfrakturen nach wie vor die exakte anatomische Reposition der Gelenkfragmente mit möglichst genauer Wiederherstellung der Gelenkkongruenz oberstes Behandlungsziel. Im Gegensatz dazu braucht es aber bei Frakturen des meta- und diaphysären Segmentes eines Röhrenknochens

Tab. 1 Indikationsspektrum für die Osteosynthese mit einer submuskulär oder subkutan eingeschobenen Platte an Femur oder Tibia

- Fraktur bei sehr engem Markkanal - Fraktur bei sehr weitem Markkanal - pathologische Fraktur, inkl. Fraktur bei hochgradiger Osteoporose

- periprothetische Fraktur (nach Hüft-TP oder Knie-TP)

- Schaftfraktur mit meta-epiphysären Frakturausläufern

- polytraumatisierter Patient mit schwerer Thoraxverletzung und Kontraindikation für eine Marknagel-Osteosynthese

- beidseitige Femurfrakturen

- Femurfraktur mit ipsilateraler Tibiakopffraktur oder Knieluxation

- assoziierte Gefäß- oder Nervenläsionen 
zur Wiederherstellung einer guten Extremitätenfunktion lediglich das korrekte Einrichten der Fraktur bezüglich der ursprünglichen Knochenlänge, der Achsen in zwei Ebenen und der Knochentorsion.

Diese Behandlungsziele lassen sich zumindest im Bereich des diaphysären Segmentes eines Röhrenknochens mit einer Marknagel-Osteosynthese meist mühelos erreichen. Da aber auch nach Marknagelung Komplikationen auftreten und in bestimmten Fällen eine Kontraindikation gegen eine Marknagel-Osteosynthese besteht, drängt sich eine Neubewertung der Osteosyntheseindikationen auf. Die Platten-Osteosynthese kennt auch im Bereich der Knochendiaphyse eine große Anzahl spezifischer Indikationen (Tab.1). Damit besteht zu Recht ein großer Bedarf, neue Plattendesigns, indirekte Repositionstechniken und schonungsvolle Stabilisierungsverfahren zu entwickeln, welche die Schädigung der periostalen Blutversorgung des Knochens bei der Platten-Osteosynthese auf ein Minimum reduzieren können $[11,12,14]$. Die Platten-Osteosynthese meta- und diaphysärer Frakturen des Ober- und Unterschenkels mit submuskulärem, respektive subkutanem Einschieben des Implantates stellt in diesem Sinne eine logische Weiterentwicklung der biologischen Osteosynthese dar [7,9]. Diese minimal-invasive Osteosynthesetechnik wird in unserem Spital seit 1995 angewendet, wir berichten im Folgenden über die Operationstechnik und die ersten klinischen Erfahrungen.

\section{Allgemeine Regeln}

In Analogie zu einer Marknagel-Osteosynthese wird die Fraktur mit der Platte langstreckig überbrückt unter korrekter Einstellung von Länge, Achsen und Torsion des Knochens. Durch den Gebrauch einer langen Platte vergrößert sich der mechanisch wirksame Hebelarm der Platte. Dadurch nimmt die Belastung der Plattenschrauben deutlich $a b$, und die Gefahr des Ausreißens einzelner Schrauben oder eines Schraubenbruchs wird vermindert. Die langstreckige Überbrückung einer Frakturtrümmerzone führt als weiteres zu einer langstreckigen Verteilung der Implantat- und Gewebebelastung. Für das Gewebe bedeutet dies eine Verkleinerung der Gewebedehnung und damit eine ungestörte Differenzierung des Kallusblastems, für das Implantat eine Verkleinerung der elastischen Deformation unter gegebener äußerer Last und damit eine Verbesserung
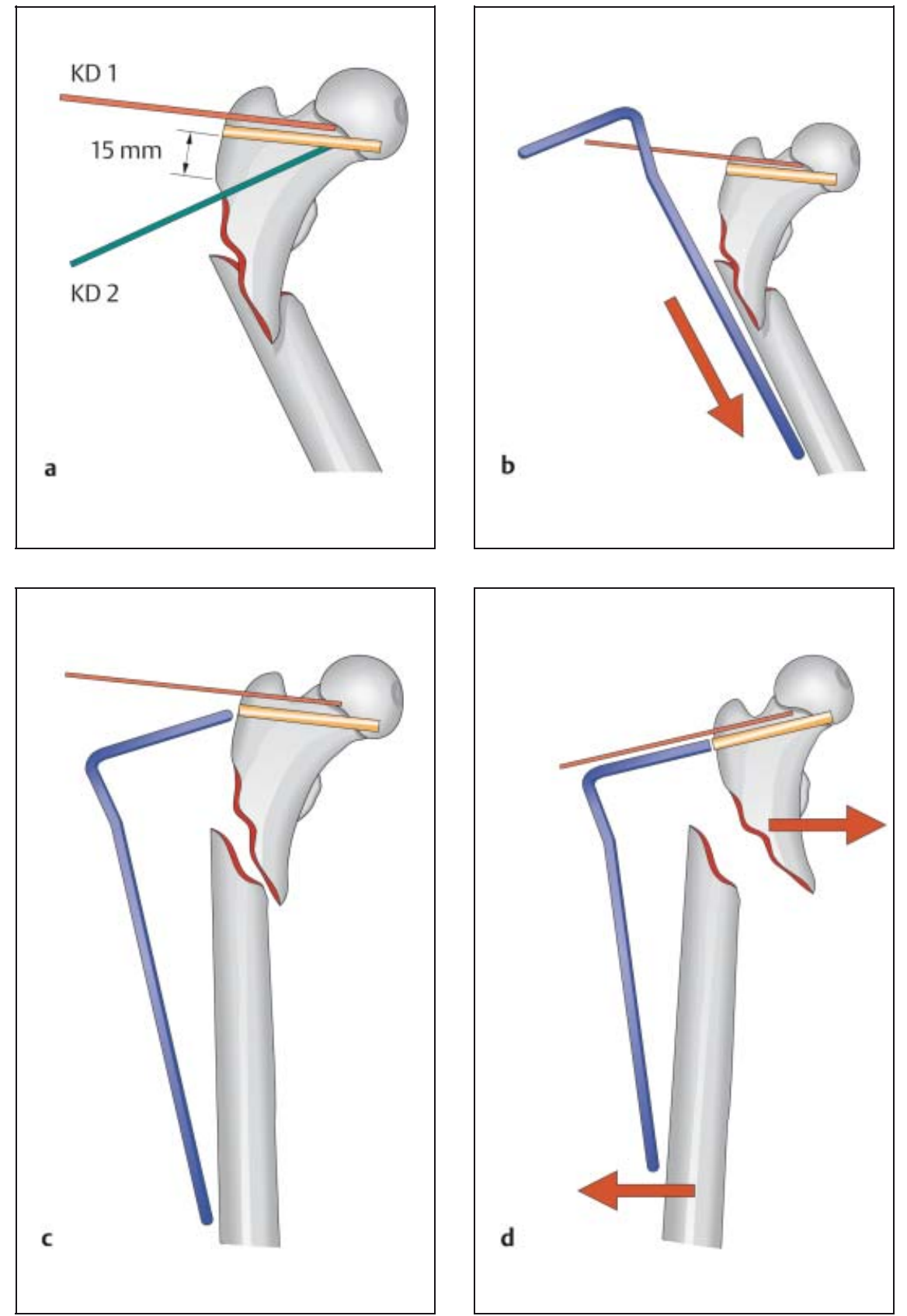

Abb.1 Ablaufschema für das submuskuläre Einbringen einer Kondylenplatte im Bereich des proximalen Femurs.

Abb.1 a Das Klingenfenster wird $15 \mathrm{~mm}$ proximal des Tuberculum innominatum angelegt und die Klingenrichtung mit einem Referenz-Kirschner-Draht (KD 1) und einem Antetorsions-Kirschner-Draht (KD 2) definiert.

Abb.1 b Die Kondylenplatte wird von proximal her unter den Vastus lateralis eingeschoben. Die Plattenklinge ist dabei nach lateral gerichtet.

Abb.1c Nach Drehen der Platte um $180^{\circ}$ (Klinge nach medial) ergibt sich als Problem eine Divergenz zwischen der Ausrichtung der Plattenklinge und der Richtung des vorbereiteten Klingensitzes.

Abb.1 d Durch Adduktion des proximalen Hauptfragmentes und Abduktion des Beines wird die notwendige Parallelität zwischen Plattenklinge und Klingensitz hergestellt, so dass sich die Klinge mühelos in den Knochen vorschieben lässt. 
des Ermüdungsverhaltens. Nach Rozbruch et al. (1998) kommt es unter dieser mechanischen Konstellation recht selten zu einer bleibenden plastischen Deformation der Platte (Plattenbiegung) durch einen einzelnen zu hohen Belastungszyklus und auch selten zum Plattenbruch durch Überschreiten der Ermüdungsfestigkeit des Implantates.

Die Platte soll im proximalen und distalen Hauptfragment durch mindestens zwei, bei Gebrauch von Titanimplantaten mit drei Schrauben fixiert werden. Am Femur ist bei sehr distal oder proximal subtrochanter liegender Fraktur zur mechanischen Balancierung der Osteosynthese der Gebrauch einer submuskulär eingeschobenen Kondylenplatte (oder eines Implantates mit winkelstabilen Schrauben, z.B. eines LISS Less Invasive Stabilization System) zu empfehlen. Sowohl bei Trümmerfrakturen als auch bei einfachen Quer- oder Schrägfrakturen empfiehlt es sich, in Plattenmitte über der Fraktur mehrere Plattenlöcher nicht mit Schrauben zu besetzen.

Folgende Repositions- und Fixationsprinzipien gilt es bei der eingeschobenen Plattenosteosynthese an Femur und Tibia zu beachten:

- keine Darstellung der Frakturzone, damit keine chirurgische Denudierung von Fragmenten

- indirekte Repositionstechnik der Fraktur mit dem Hauptaugenmerk auf eine korrekte Längen-, Achsen- und Torsionseinstellung

- Stabilisierung der Fraktur ohne interfragmentäre Kompression (keine freien Zugschrauben, keine Plattenzugschrauben, kein Spanner, keine exzentrischen Plattenschrauben)

- Gebrauch einer Platte mit verminderter Kontaktfläche (LC-DCP: Limited Contact Dynamic Compression Plate) oder eines Implantates mit winkelstabilen Schrauben (LISS: Less Invasive Stabilization System, LCP: Locking Compression Plate)

- Titan als Implantatmaterial

- keine primäre Spongiosaplastik

\section{Operationstechnik}

\section{Technik am proximalen Femur}

Das proximale Femur wird über einen antero-lateralen Zugang nach Watson-Jones exponiert. Ist die Verwendung einer Kondylenplatte geplant, so empfiehlt es sich, das Hüftgelenk chirurgisch zu eröffnen und zur sicheren Positionierung der Klinge den medial distalen Femurkopfquadranten darzustellen. Das Klingenfenster
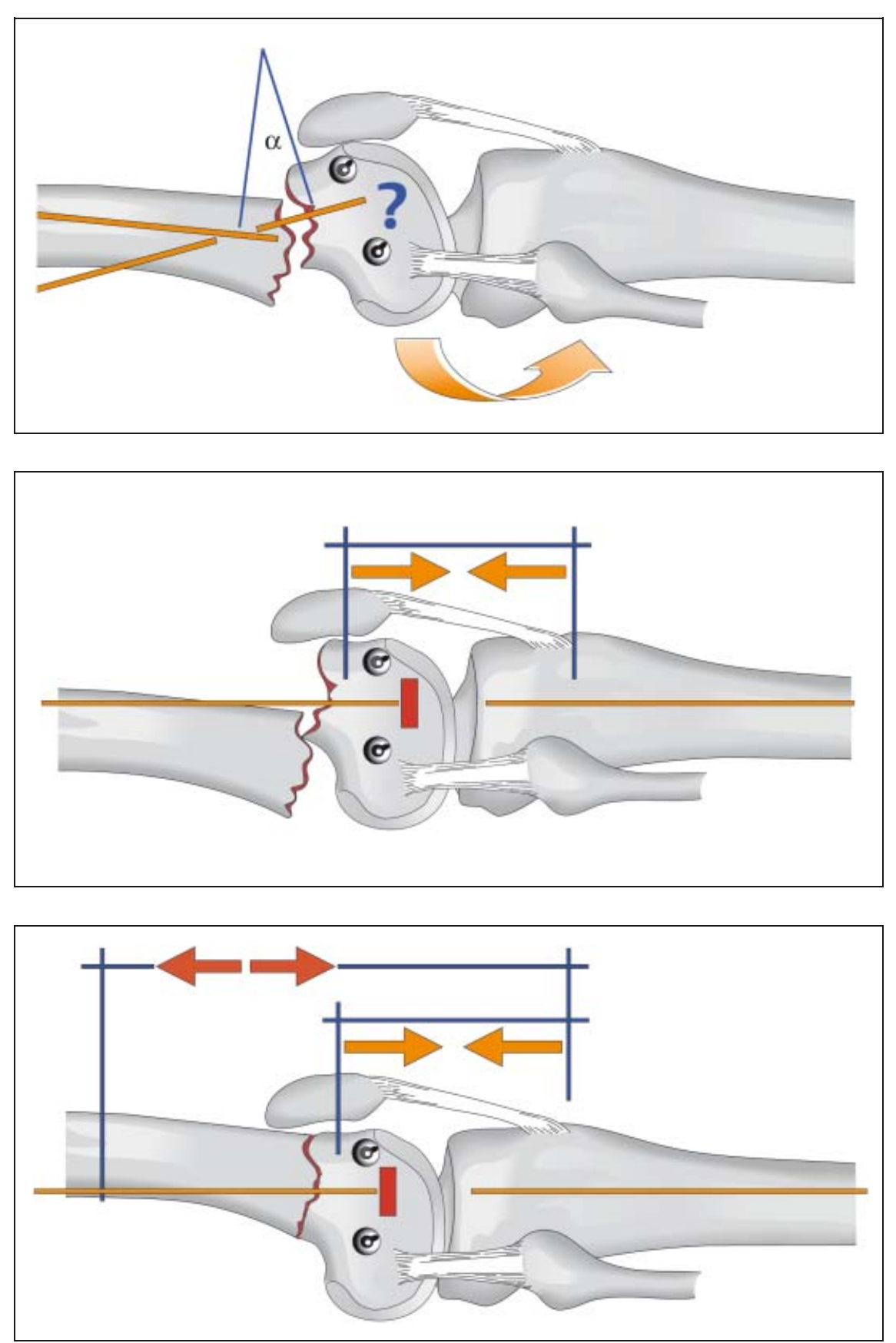

Abb. 2 Schema der korrekten Klingeninsertion im Bereich des distalen Femurs.

Abb. 2a Bei einer distalen Femurfraktur findet sich der Gelenkblock durch den Zug der Muskulatur (Gastrocnemii) meist in einer Extensionsfehlstellung. Bei kurzem distalen Fragment stellt deswegen die korrekte Ausrichtung des Klingensitzes bezüglich der Flexion-Extension das Hauptproblem dar.

Abb.2b Es ist deswegen von Vorteil, den distalen Gelenkblock mittels eines Joysticks in volle Extensionsstellung im Kniegelenk zu bringen und mit Kirschner-Drähten oder einem ventralen Fixateur externe in dieser Position zu fixieren. Damit ergibt die Verlängerung der Unterschenkelachse nach proximal die korrekte Klingenausrichtung bezüglich Flexion-Extension.

Abb.2c Die Platte wird nachfolgend von distal her submuskulär eingeschoben. Die definitive Reposition von Länge und Achsen wird mit Hilfe eines Distraktors durchgeführt. 

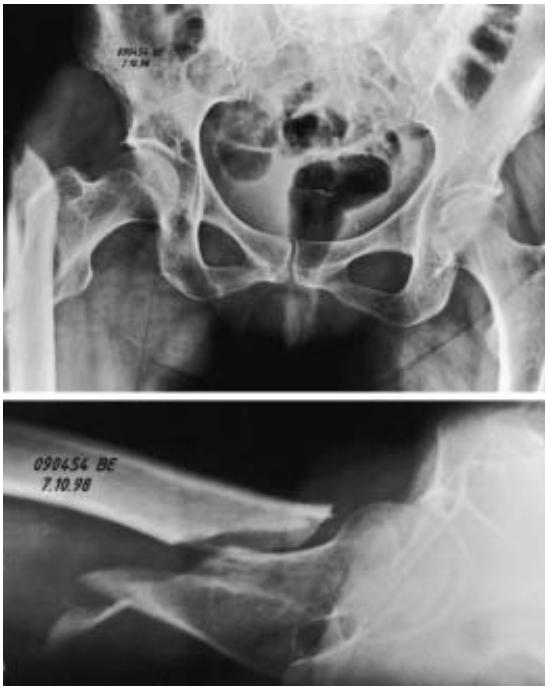

Abb.3a Dislozierte subtrochantere Femurfraktur rechts bei einer 44-jährigen Patientin.

wird $15 \mathrm{~mm}$ proximal des Tuberculum innominatum angelegt. Anschließend werden ein Antetorsions-KirschnerDraht und ein Referenz-Kirschner-Draht in geplanter Klingenrichtung eingebracht und der Klingensitz vorbereitet (Abb.1 a). Die Platte wird dann submuskulär eingeschoben, wobei zur Schonung des Vastus lateralis die Platte um $180^{\circ}$ verdreht, d.h. mit der Klinge nach außen zeigend, eingebracht wird (Abb.1b). Die Platte wird sodann um $180^{\circ}$ gedreht, so dass die Klinge nach medial weist. Um die Klinge gefahrlos einzubringen, muss zuerst durch Abduktion des Beines oder Adduktion des proximalen Hauptfragmentes mit einem Joystick eine korrekte parallele Ausrichtung zwischen der Plattenklinge und dem vorbereiteten Klingensitz, respektive dem Referenz-Kirschner-Draht erzielt werden (Abb.1 c). Erst danach lässt sich die Klinge mühelos manuell in den Knochen eindrücken. Die Fraktur wird indirekt mit Hilfe eines Distraktors reponiert und die Plattenschrauben perkutan eingebracht (Abb.1d). Die erreichte Reposition wird vorgängig unter Bildwandler (Achsen, Länge) und klinisch (balancierte Rotation der Hüfte) geprüft.

\section{Technik am distalen Femur}

Das distale Femur wird über einen kurzen lateralen Subvastuszugang dargestellt. Es erfolgt die laterale Arthrotomie und die Schraubenosteosynthese einer allfällig vorhandenen Gelenkfraktur. Bei Verwendung einer Kondylenplatte liegt das Klingenfenster rund $10 \mathrm{~mm}$ posterior der ventralen Kondylenbegrenzung und

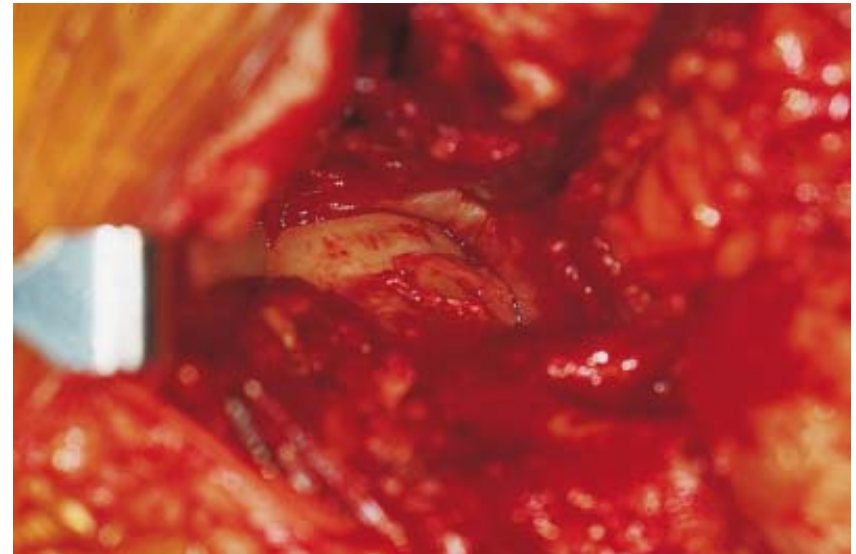

Abb. 3b Nach ventraler Arthrotomie des Hüfte Darstellen des medial unteren Femurkopfquadranten zum sicheren Einbringen der Plattenklinge.

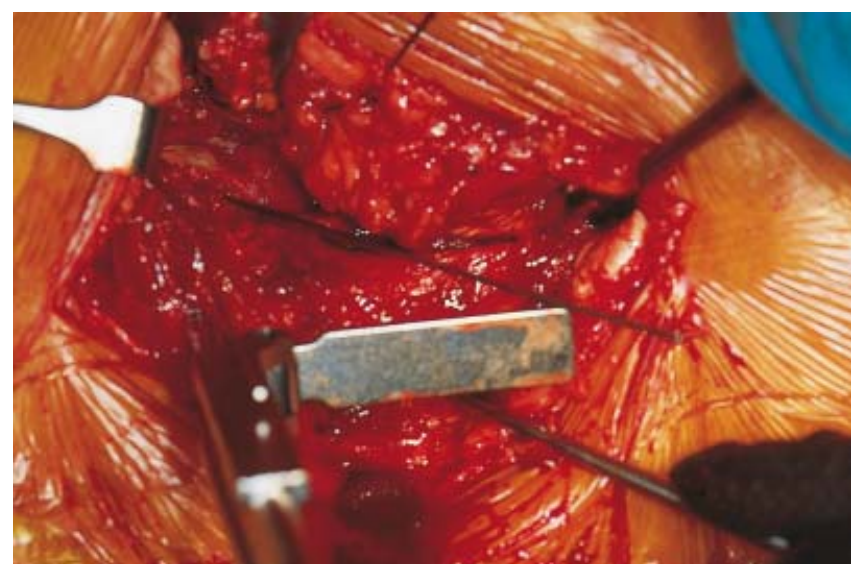

Abb. 3c Einbringen des AntetorsionKirschner-Drahtes und Präparation des Klingensitzes mit dem Standard-Instrumentarium.

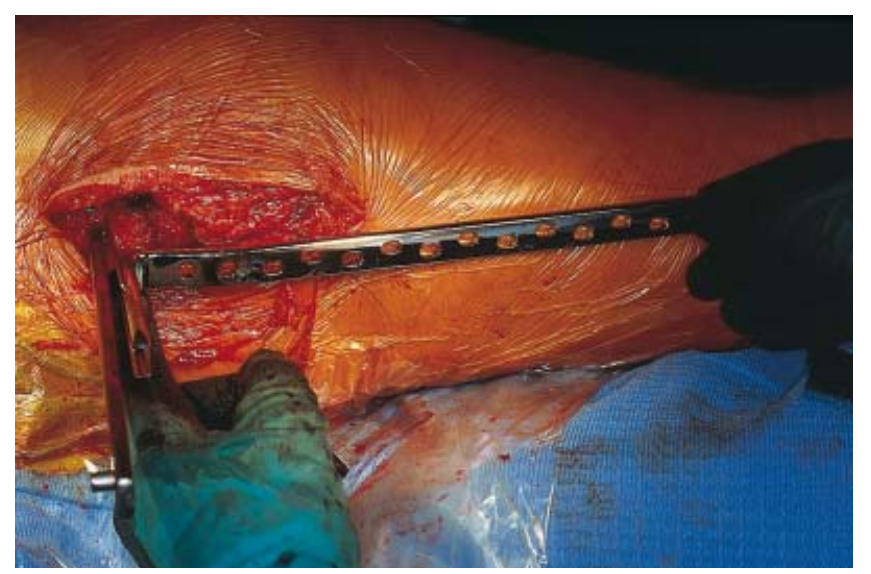

Abb.3d Vergleich zwischen der Länge der Hautinzision und der Plattenlänge.

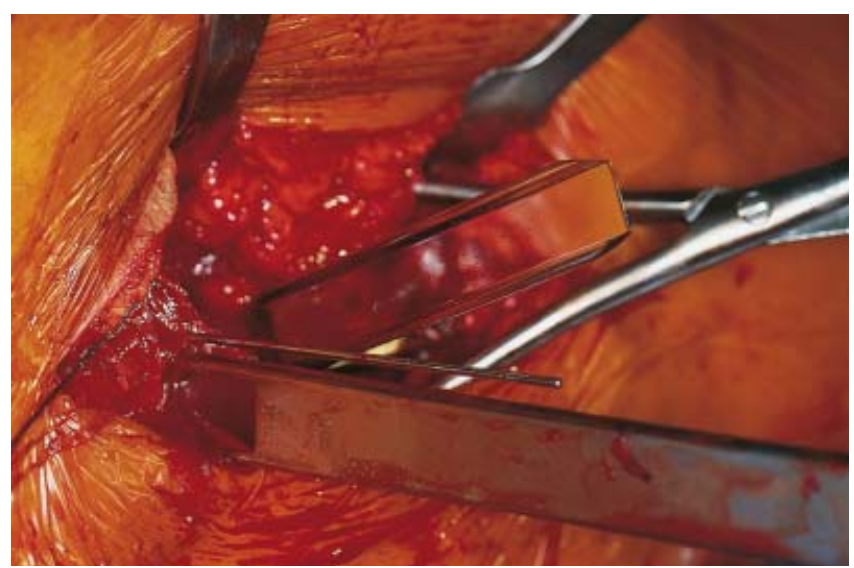




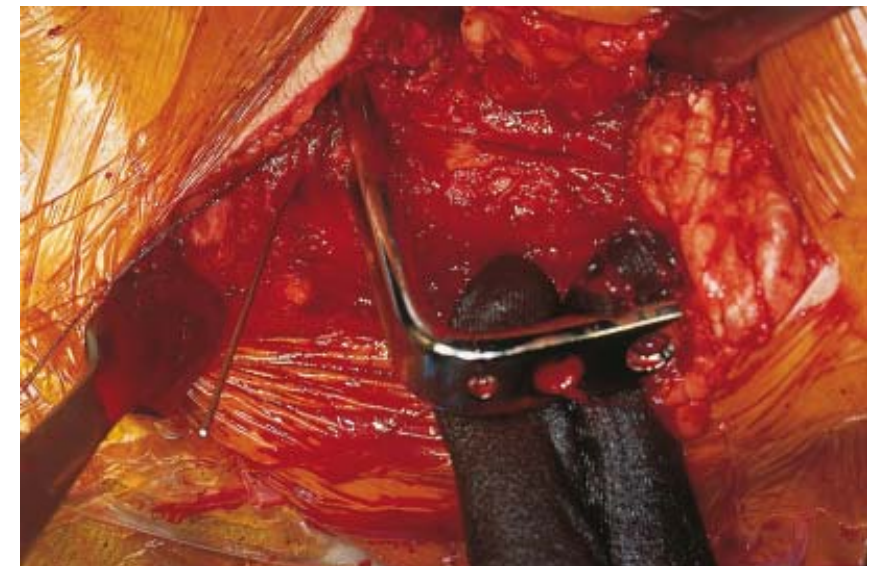

Abb. $3 \mathbf{f}$ Entfernung des Klingensitz-Instrumentes und Drehen der Platte um $180^{\circ}$. Sichtbare Konvergenz zwischen proximal liegendem Referenz-Kirschner-Draht (Richtung des Klingensitzes) und Plattenklinge. Erreichen der Parallelität zwischen Klingensitz und Plattenklinge durch Adduktion des proximalen Fragmentes und Abduktion des distalen Hauptfragmentes.

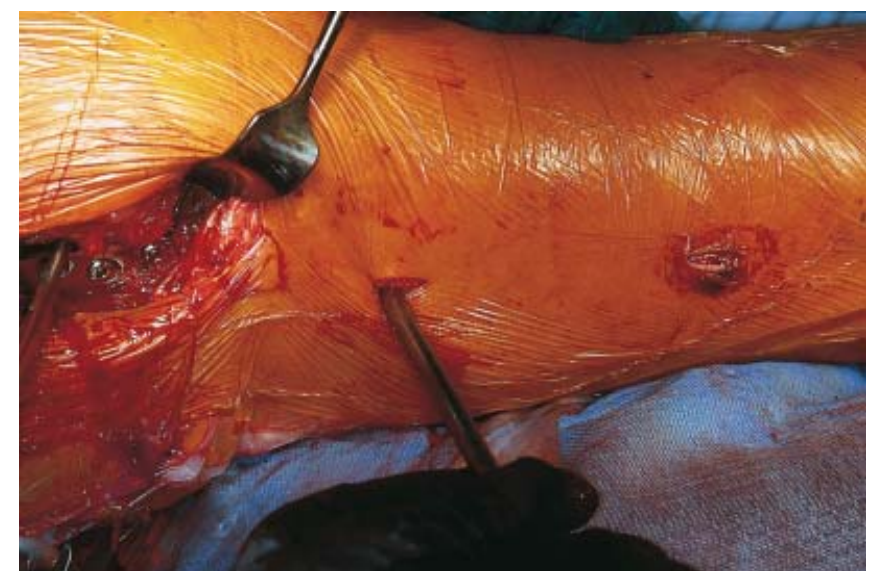

Abb.3g Perkutanes Einbringen von Plattenschrauben.

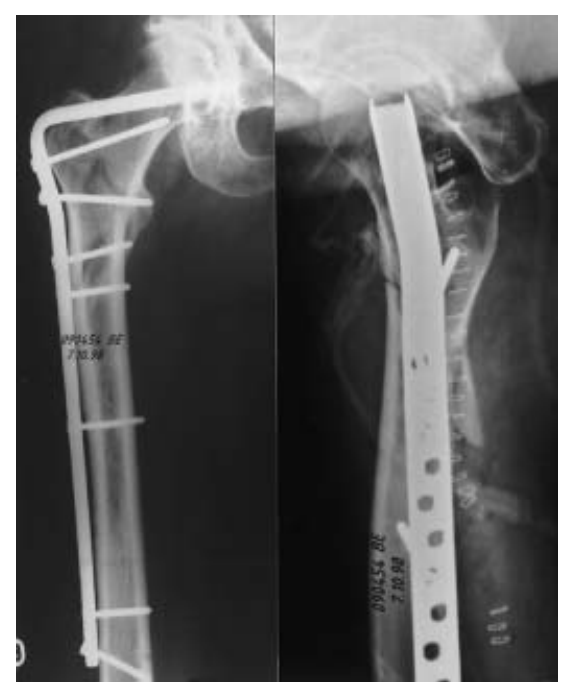

Abb. 3h Postoperatives Röntgenbild mit gutem Alignement der Fraktur in beiden Ebenen.

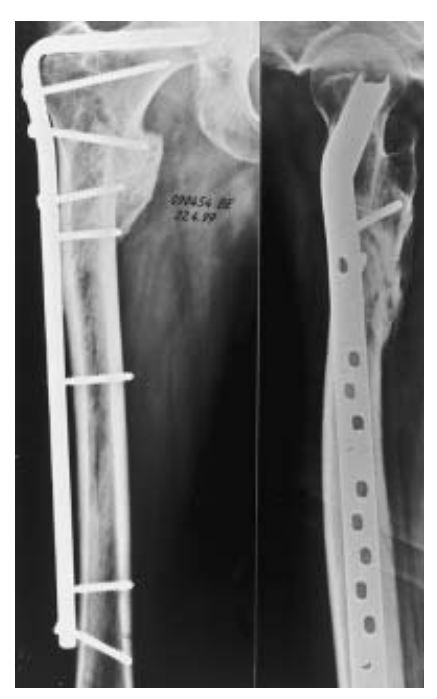

Abb. $\mathbf{3 i}$ Konsolidierung der Fraktur in achsengerechter Stellung. rund $15 \mathrm{~mm}$ proximal des Kniegelenkspaltes. Der Klingensitz muss parallel zum femoro-tibialen Gelenkspalt und mit Vorteil orthograd zur lateralen Kondylenwange angelegt werden. Bei einem sehr kurzen distalen Gelenkblock liegt die Hauptschwierigkeit indessen in der korrekten Flexions-Extensions-Orientierung des Klingensitzes (Abb. 2a). Es empfiehlt sich deswegen, das distale Hauptfragment mit einem Joystick in volle Extensionsstellung bezüglich des Kniegelenkes zu bringen und mit KirschnerDrähten oder einem ventralen Fixateur präliminär zu stabilisieren (Abb. $\mathbf{2 b}$ ). Damit wird der Zug der Gastrocnemii neutralisiert und die Unterschenkelachse gibt in seiner Verlängerung nach proximal die korrekte Flexions-ExtensionsEinstellung des Klingenfensters an (Abb.2c). Die Platte wird submuskulär nach proximal geschoben, wobei auch hier die Klinge initial nach lateral gerichtet ist. Nachfolgend wird die Klinge um $180^{\circ}$ gedreht, der Unterschenkel zusammen mit dem distalen Femur abduziert und die Klinge ins Kondylenmassiv eingebracht. Die Reposition erfolgt indirekt, die Repositionskontrolle unter Bildwandler und bezüglich der Torsion auch klinisch.

\section{Technik an der distalen Tibia}

Die Fraktur wird zuerst manuell grob reponiert, und der Innenknöchel über eine rund $4 \mathrm{~cm}$ lange Inzision epiperiostal dargestellt. Anschließend erfolgt das stumpfe Eröffnen des Subkutanraumes, sei es direkt mit der Platte, sei es mit einem speziellen schuhlöffelartigen Instrument. Die Platte wird entsprechend der Krümmung der medialen Tibiakortikalis vorgebogen. Sofern eine eher dorsale Plattenlage gewählt wird, erübrigt sich ein zusätzliches Verwinden der Platte. Die Platte wird von distal her subkutan eingeschoben, was meist bereits zu einer recht guten Einstellung von Fraktur und Unterschenkelachsen führt. Die Höhe des Gelenkspaltes des oberen Sprunggelenkes wird mittels eines Kirschner-Drahtes unter Bildwandler definiert und die erste Plattenschraube möglichst weit distal eingebracht. Nachfolgend erfolgt die definitive Reposition der Fraktur. Die Platte wird perkutan fixiert und das Repositionsergebnis sowie die Lage der Implantate unter Bildwandler kontrolliert. Bei Unterschenkelfrakturen mit intraartikulären Frakturausläufern oder bei sehr distal liegender Fibulafraktur empfiehlt es sich, vorgängig eine zusätzliche konventionelle Plattenosteosynthese der Fibula durchzuführen. 


\section{Kasuistik und klinische Fallbeispiele}

\section{Eingeschobene Femurplatte}

Zwischen Januar 1997 und Februar 1999 wurden 20 Femurfrakturen mit einer eingeschobenen Platte stabilisiert (Tab.2). Die Frakturen des proximalen und distalen Schaftdrittels wurden ausnahmslos mit einer Kondylenplatte, die des mittleren Schaftdrittels mit einer LC-DCP versorgt. Dabei wurden 12- bis 20-Loch-Platten verwendet (Abb.3a-i). 18 Frakturen heilten in achsengerechter Stellung innerhalb von 4 Monaten aus, bei zwei Frakturen kam es aufgrund einer Heilungsverzögerung zu einem Plattenbruch - einmal 5 Monate nach Versorgung einer periprothetischen Fraktur (Hüfttotalprothese) und einmal 8 Monate nach Osteosynthese einer distalen $3^{\circ}$-offenen $\mathrm{Fe}$ murfraktur. Beide Frakturen heilten nach Reosteosynthese mittels einer Kondylenplatte und lokaler Spongiosaplastik folgenlos aus. Damit ergibt sich eine Erfolgsrate, d.h. Konsolidation der Fraktur in achsengerechter Stellung nach der ersten Operation von $18 / 20$ oder $90 \%$.

\section{Eingeschobene Tibiaplatte}

Zwischen September 1997 und Mai 1999 wurden 25 Unterschenkelfrakturen bei 24 Patienten mit einer von distal eingeschobenen Tibiaplatte versorgt (Tab.3). Zur Stabilisierung wurde eine DCP oder eine LC-DCP mit zwischen 9 und 16 Plattenlöchern verwendet. In 11 Fällen war eine zusätzliche Stabilisierung der Fibula notwendig (Abb.4a-g).

Alle Frakturen waren nach 4 Monaten konsolidiert. In zwei Fällen wurde ein tiefer Infekt (bei beiden wurde noch keine antiseptische Abdeckfolie verwendet) und in einem Fall ein postoperatives Kompartment-Syndrom beobachtet. Die Infekte heilten nach lokalem Debridement, vorzeitiger Metallentfernung und systemischer Antibiotikagabe, das Logensyndrom des Unterschenkels nach Fasziotomie folgenlos aus. In einem Fall wurde ein leichter Repositionsverlust von $4^{\circ}$ Rekurvatum beobachtet.

\section{Zusammenfassung und Schlussfolgerungen}

Bei der submuskulären Plattenosteosynthese am Femur und der subkutanen Plattenosteosynthese an der Tibia handelt es sich um eine Weiterentwicklung und Perfektionierung der biologischen Osteosynthese. Ziel des Verfahrens ist
Tab. 2 Kasuistik: eingeschobene Femurplatte

$\begin{array}{lc}\text { Patienten } & 20 \\ \text { Frauen } & 11 \\ \text { Männer } & 9 \\ \text { Alter (Jahre) } & 62 \text { (12-94) } \\ \text { Frakturlokalisation } & \\ \text { - subtrochanter } & 10 \\ \text { - mittleres Schaftdrittel } & 3 \\ \text { - distales Drittel, extra- } & 3 \\ \quad \text { artikulär } & \\ \text { - periprothetisch nach } & 4 \\ \quad \text { Hüft-TP } & \\ \text { - geschlossen } & 19 \\ \text { - offen 3 } & 1 \\ \text { Osteosynthese } & \\ \text { - Kondylenplatte proximal } & 10 \\ \text { - Kondylenplatte distal } & 6 \\ \text { - LC-DCP } & 4 \\ \text { Follow-up (Monate) } & 18(15-32) \\ \text { normale Heilung } & 18 \\ \text { Implantatbruch } & 2 \\ \text { Infekt } & 0 \\ \text { Erfolgsquote } & 18 / 20 \quad 90 \% \\ \text { Misserfolgsquote } & 2 / 20 \quad 10 \% \\ & \end{array}$

die grobe Wiederherstellung der Knochenanatomie bezüglich Länge, Achsen und Torsion des Knochens. Dem Erhalten der Knochen- und Weichteilvaskularität wird mehr Gewicht beigemessen als dem Erzielen einer möglichst hohen primären Osteosynthesestabilität.

Vorteil der Methode ist auf der biologischen Seite die Schonung der Vaskularität des Knochens und der Weichteile im Bereich der Frakturzone.

Das Einschieben einer Platte mit nur geringem Knochenkontakt beinhaltet eine nur geringfügige biologische Störwirkung. Die Erhaltung der biologischen Kompetenz aller involvierten Gewebe hat eine weitgehend natürliche Frakturheilung zur Folge, die Konsolidation erfolgt meist über einen Fixationskallus und das nachfolgende intrakortikale Remodelling stellt die ursprüngliche Knochenstruktur, -steifigkeit und -festigkeit wieder her. Am Femur stellt der Umstand, dass der Vastus lateralis nicht weitstreckig vom Femurschaft abgelöst werden muss, bezüglich postoperativer Lagerung und schneller Rehabilitation einen zusätzlichen Vorteil gegenüber der offenen Osteosynthesetechnik dar.

Auf der mechanischen Seite überbrückt die Osteosyntheseplatte als Kraftträger ähnlich einem Marknagel die Frakturzone, Unterschied ist einzig die extramedul-
Tab. 3 Kasuistik: eingeschobene Tibiaplatte

\begin{tabular}{lc} 
Frakturen & 25 \\
Patienten & 24 \\
Frauen & 13 \\
Männer & 11 \\
Alter (Jahre) & 42 (16-64) \\
Frakturlokalisation & \\
- diaphysär & 19 \\
- distal metaphysär & 4 \\
- distal intraartikulär & 2 \\
- geschlossen & 22 \\
- offen 1 & 3 \\
Osteosynthese & \\
- DCP 4.5 Stahl & 1 \\
- LC-DCP 4.5 & 24 \\
- Tibiaosteosynthese allein & 14 \\
- Fibula-Osteosynthese & 11 \\
- Volkmann-Osteosynthese & 2 \\
Follow-up (Monate) & 18 (10-27) \\
normale Heilung & 23 \\
Implantatbruch & 0 \\
Infekt & 2 \\
Kompartmensyndrom & 1 \\
Erfolgsquote & $23 / 2592 \%$ \\
Misserfolgsquote & $2 / 258 \%$ \\
\hline
\end{tabular}

läre Lage der Platte gegenüber der intramedullären Lage des Marknagels. Mechanisch gesehen ist die langstreckige Überbrückung der Fraktur wichtig, da dadurch sowohl die lokale Plattenbelastung im Frakturbereich (elastische Deformation unter äußerer Last) als auch die Belastung der Plattenschrauben abnehmen. Damit verringert sich die Gefahr des Auftretens rein mechanischer Komplikationen wie Schraubenausrisse, Implantatbrüche oder Implantatbiegung. Die mechanische Leistungsfähigkeit des Implantates wird damit optimal eingesetzt.

Nachteil der minimal-invasiven Osteosynthesetechnik ist die schwierigere, da indirekte Repositionstechnik, die Verwendung des Bildwandlers zur Repositionskontrolle und ein noch nicht ausgereifter Implantat- und Instrumentsatz.

Trotzdem lässt sich diese Technik bereits jetzt unter Verwendung von gängigen Standard-Implantaten anwenden und es lassen sich im Vergleich zur MarknagelOsteosynthese vergleichbar gute Resultate erzielen. Neben einer sorgfältigen und atraumatischen Operationstechnik sind eine korrekte Patientenselektion mit genauer Evaluation eines allfällig begleitenden Weichteilschadens und eine vorsichtige funktionelle Nachbehandlung bis zur radiologisch gesicherten Konsolidation des Knochens wichtige Elemente zum Behandlungserfolg. 

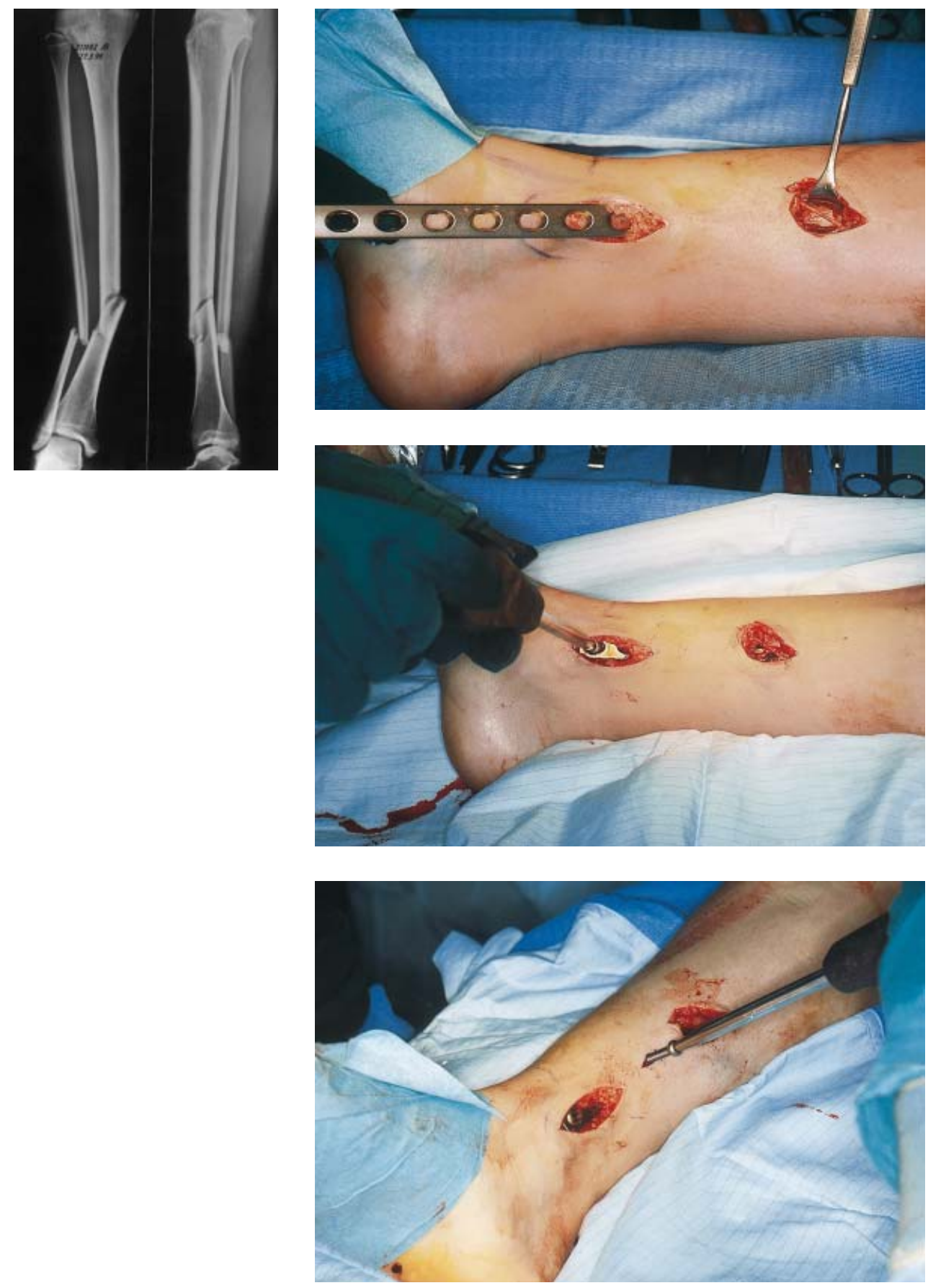

Abb. 4a $\quad 1^{\circ}$-offene Unterschenkelfraktur rechts bei einer 16 -jährigen Patientin.

Abb. 4b Subkutanes Einschieben der LC-DCP von distal her über eine kurze Längsinzision im Bereich des Innenknöchels. Vorgängiges Weichteil-Débridement einer Hautdurchspießung über der Fraktur.

Abb.4c Einbringen der distalen Plattenschraube.

Abb.4d Perkutanes Einbringen weiterer Plattenschrauben.

Abb.4e Postoperatives Röntgenbild mit perfekter Reposition in beiden Ebenen und korrekter Lage des Osteosynthesematerials.

Abb.4f Ausheilungsbild 1,5 Jahre nach Osteosynthese.

Abb.4g Vollständig normale Knochenstruktur nach Metallentfernung. 
Eine weitere Vereinfachung dieser minimal-invasiven Operationstechniken wird mit weiteren Neuentwicklungen auf dem Implantatsektor - wie zum Beispiel anatomisch vorgeformten Platten (LISS, Less Invasive Stabilization System), Platten mit winkelstabilen Plattenschrauben (LCP, Locking Compression Plate) oder neuartigen selbstbohrenden und/oder selbstschneidenden Schrauben - zu erwarten sein. Neue Instrumente, Hilfsmittel und auch Computersysteme (CAOS, computerassistierte orthopädische Chirurgie) zur Erleichterung der indirekten Reposition sowie zur Repositionskontrolle sind in Planung und auch bereits in klinischer Evaluation, so dass unsere heute noch gültigen Ziele und Methoden von Reposition und Stabilisation im Rahmen der operativen Frakturversorgung weiter revolutioniert werden dürften.

\section{Literatur}

${ }^{1}$ Arens S, Kraft C, Schlegel U et al. Susceptibility to local infection in biological internal fixation. Experimental study of open vs minimally invasive plate osteosynthesis in rabbits. Arch. Orthop. Trauma Surg. 1999; 119: 82-85

2 Farouk O, Krettek C, Miclau Tet al. Minimally invasive plate osteosynthesis: does percutaneous plating disrupt femoral blood supply less than the traditional technique? J. Orthop. Trauma 1999; 13: $401-406$

${ }^{3}$ Gautier E, Cordey J, Lüthi UK et al. Knochenumbau nach Verplattung - Biologische oder mechanische Ursache? Hefte Unfallheilkd. 1983; 165: $13-15$

${ }^{4}$ Gautier E, Perren SM, Ganz R. Principles of internal fixation. Curr. Orthop. 1992; 6: 220-232

${ }^{5}$ Gautier E, Rahn BA, Perren SM. Vascular remodelling. Injury 1995; 26 Suppl 2: 11-19

${ }^{6}$ Gerber C, Mast JW, Ganz R. Biological internal fixation of fractures. Arch. Orthop. Trauma Surg. 1990; 109: 295-303

${ }^{7}$ Helfet DL, Shonnard PY, Levine D et al. Minimally invasive plate osteosynthesis of distal fractures of the tibia. Injury 1997; 28 Suppl 1: $42-48$

${ }^{8}$ Klein MPM, Frigg R, Kessler $S$ et al. Reaming versus non-reaming in medullary nailing. Arch. Orthop. Trauma Surg. 1990; 109: $314-316$

9. Krettek C, Schandelmaier P, Tscherne H. Distale Femurfrakturen. Unfallchirurg 1996; 99 : $2-10$

${ }^{10}$ Lüthi UK, Frigg R, Stroud RD. Implantate und Knochenreaktion in vivo. Hefte Unfallheilkd. 1983; 165: 5-6

${ }^{11}$ Mast JW, Jakob RP, Ganz R. Planning and reduction technique in fracture surgery. Springer, Berlin, Heidelberg, New York (1989)

12 Miclau T, Martin RE. The evolution of modern plate osteosynthesis. Injury 1997; 28 Suppl 1: 3-6
${ }^{13}$ Müller ME, Allgöwer M, Schneider R, Willenegger $\mathrm{H}$. Manual der Osteosynthese. Springer, Berlin, Heidelberg, New York (1977)

14 Perren SM. The concept of biological plating using the limited contact dynamic compression plate (LC-DCP). Injury 1991; 22 Suppl 1: $1-41$

15 Pfister U, Rahn BA, Perren SM et al. Vaskularität und Knochenumbau nach Marknagelung langer Röhrenknochen. Aktuel. Traumatol. 1979; 9: 191 - 195

${ }^{16}$ Rozbruch SR, Müller U, Gautier E et al. The evolution of femoral shaft plating technique. Clin. Orthop. 1998; 354: 195-208

${ }^{17}$ Weller S, Höntzsch D, Frigg R. Die epiperostale, perkutane Plattenosteosynthese. Eine minimal-invasive Technik unter dem Aspekt der „biologischen Osteosynthese“. Unfallchirurg 1998; 101: 115-121

${ }^{18}$ Wenda K, Runkel M, Degreif J et al. Minimally invasive plate fixation in femoral shaft fractures. lnjury 1997; 28 Suppl 1: 13-19

Dr. med. E. Gautier

stellvertretender Chefarzt

Dr. med. C. B. Marti

Oberarzt

Dr. med. A. J. Schuster

Oberarzt

Dr. med. S. W. Wachtl

Oberarzt

Prof. Dr. med. R. P. Jakob

Chefarzt

Orthopädische Klinik

Kantonsspital

CH-1708 Fribourg

Schweiz 